\title{
Hippocampus Glutamate and N-Acetyl Aspartate Markers of Excitotoxic Neuronal Compromise in Posttraumatic Stress Disorder
}

\author{
Isabelle M Rosso*, 1,2 , David J Crowley', Marisa M Silveri ${ }^{2,3}$, Scott L Rauch ${ }^{1,2}$ and J Eric Jensen ${ }^{2,3}$ \\ 'Center for Depression, Anxiety and Stress Research, McLean Hospital, Belmont, MA, USA; '2Department of Psychiatry, Harvard Medical School, \\ Boston, MA, USA; ${ }^{3}$ McLean Imaging Center, McLean Hospital, Belmont, MA, USA
}

\begin{abstract}
Hippocampus atrophy is implicated in posttraumatic stress disorder (PTSD), and may partly reflect stress-induced glutamate excitotoxicity that culminates in neuron injury and manifests as re-experiencing symptoms and other memory abnormalities. This study used high-field proton magnetic resonance spectroscopy (MRS) to determine whether PTSD is associated with lower hippocampus levels of the neuron marker $\mathrm{N}$-acetyl aspartate (NAA), along with higher levels of glutamate (Glu) and Glu/NAA. We also predicted that metabolite levels would correlate with re-experiencing symptoms and lifetime trauma load. Twenty-four adult PTSD patients and 23 trauma-exposed normal controls (TENC) underwent 4T MRS of the left and right hippocampus. Participants received psychiatric interviews, and completed the Traumatic Life Events Questionnaire to define lifetime trauma load. Relative to TENC participants, PTSD patients exhibited significantly lower NAA in right and left hippocampi, and significantly higher Glu and Glu/NAA in the right hippocampus. Re-experiencing symptoms were negatively correlated with left and right NAA, and positively correlated with right Glu and right Glu/NAA. Trauma load was positively correlated with right Glu/NAA in PTSD patients. When re-experiencing symptoms and trauma load were examined together in relation to right Glu/NAA, only re-experiencing symptoms remained a significant correlate. This represents the first report that PTSD is associated with MRS markers of hippocampus Glu excess, together with indices of compromised neuron integrity. Their robust associations with re-experiencing symptoms affirm that MRS indices of hippocampus neuron integrity and glutamate metabolism may reflect biomarkers of clinically significant disease variation in PTSD.

Neuropsychopharmacology (2017) 42, 1698-1705; doi:I0.1038/npp.2017.32; published online 8 March 2017
\end{abstract}

\section{INTRODUCTION}

Posttraumatic stress disorder (PTSD) involves persistent emotional reactivity to trauma reminders in environments where a threat is no longer present. One robust neurobiological correlate of PTSD is a smaller size of the hippocampus (Woon et al, 2010), a brain region that is critical for distinguishing safe from dangerous contexts (Rauch et al, 2006) and that is particularly susceptible to stress-induced morphological alterations (McEwen, 2007). Since publication of the seminal magnetic resonance imaging (MRI) report of hippocampal atrophy in PTSD (Bremner et al, 1995), metaanalyses have confirmed smaller bilateral hippocampus volumes in patients (Karl et al, 2006; Kitayama et al, 2005; Smith, 2005; Woon et al, 2010).

Multiple lines of evidence point to glutamate excitotoxicity as a contributor to hippocampus neuron injury in PTSD

* Correspondence: Dr IM Rosso, CDASR, McLean Hospital, Harvard Medical School, I 5 Mill Street, Mailstop 334, Belmont, MA 02478, USA, Tel: +617 855 2607, Fax: +617 855 37।3,

E-mail: irosso@hms.harvard.edu

Received I5 September 2016; revised 23 December 2016; accepted 3 February 2017; accepted article preview online 13 February 2017
(Bremner et al, 2004; McEwen, 2006; Woon et al, 2010). Glutamate excitotoxicity has been implicated as a mechanism leading to neuron death in several neurological disorders, including amyotrophic lateral sclerosis (ALS), mesial temporal sclerosis, multiple sclerosis, and epilepsy (Lewerenz and Maher, 2015; Mark et al, 2001). In animal models of psychological trauma, exposure to repeated stress causes dendritic atrophy of pyramidal neurons in the hippocampus (Adamec et al, 2012; McEwen and Magarinos, 1997; McEwen, 2007; Popoli et al, 2012; Sapolsky, 1996), and this is mediated partly by glutamate excitotoxicity (McEwen, 2007). Glutamatergic alterations that occur during stress can produce deficits in associative learning and memory (Chambers et al, 1999; Krystal et al, 1994). Altogether, this suggests that markers of glutamate metabolism and excitotoxicity may be relevant to hippocampus neuron compromise and memory-related (re-experiencing) symptoms in PTSD.

Advances in magnetic resonance spectroscopy (MRS) have led to reliable quantification of glutamate metabolism in the brain. At lower field strengths (eg, 1.5T), the combination of glutamate and glutamine ('Glx') can be assessed reliably, while at higher fields $(\geqslant 3 \mathrm{~T})$ the glutamate $(\mathrm{Glu})$ and 
glutamine (Gln) resonances can be reliably separated (Jensen et al, 2009). In mesial temporal sclerosis, glutamate excitotoxicity manifests as elevated Glx peaks (Mark et al, 2001). In epilepsy and multiple sclerosis, elevated levels of Glx and Glu normalized to creatine (Cr) have been reported in subcortical nuclei (Doelken et al, 2010; Helms et al, 2006; Srinivasan et al, 2005; Tisell et al, 2013), and are accompanied by lower levels of the neuron marker $\mathrm{N}$-acetyl aspartate (NAA). Similarly, both $\mathrm{Glx} / \mathrm{Cr}$ and Glx/NAA are higher in epileptogenic medial temporal cortex than in the contralateral non-epileptogenic region (Savic et al, 2000), and these neurochemical differences are used as indicators of neurotoxicity. Finally, MRS studies of ALS have identified high Glu and low NAA in the motor cortex, a profile consistent with glutamate excitotoxicity hypotheses of ALS pathogenesis (Jing and Lin, 2010). Altogether, this literature identifies high Glu ratios and low NAA ratios, as well as high Glu/NAA, as MRS biomarkers of glutamate neurotoxicity.

About a dozen MRS investigations have examined hippocampus neuron integrity in PTSD, and most have found lower NAA in patients compared with traumaexposed and non-traumatized comparison subjects (Karl and Werner, 2010). A meta-analysis confirmed lower bilateral hippocampus NAA in PTSD (Karl and Werner, 2010), corresponding to medium effect sizes when NAA was normalized to water and small-to-medium effect sizes when normalized to Cr. One gap in this literature is that less than half of studies examined relationships with illness severity. Among those that that did, most only examined total PTSD symptom scores and not subscale scores that might have dissociable biological substrates. Thus, it is unclear to what extent hippocampus NAA deficits scale with PTSD symptom clusters, and/or with trauma load. It is also unknown whether lower hippocampus NAA co-occurs with higher glutamate, as prior MRS studies have not concurrently reported on NAA and Glu (or Glx). With the exception of

Table I Demographic and Clinical Characteristics of Posttraumatic Stress Disorder (PTSD) and TENC Participants

\begin{tabular}{lcc}
\hline Characteristic & PTSD $(\boldsymbol{n}=\mathbf{2 4})$ & TENC $(\boldsymbol{n}=\mathbf{2 3})$ \\
\hline Age, years & $36.49 \pm 12.33$ & $35.67 \pm 12.16$ \\
Education, years & $15.32 \pm 2.45$ & $16.38 \pm 1.47$ \\
Sex, female & $16(67)$ & $16(70)$ \\
Handedness, right & $24(100)$ & $23(100)$ \\
TLEQ, total score ${ }^{\text {a }}$ & $7.63 \pm 3.76$ & \\
CAPS, total symptoms & $59.46 \pm 23.83 \pm 1.52$ \\
CAPS, re-experiencing & $18.79 \pm 7.01$ & \\
CAPS, hyperarousal & $17.25 \pm 9.53$ & \\
CAPS, avoidance & $23.42 \pm 11.24$ & \\
Medications & & \\
None & $21(88)$ & \\
Sertraline & $2(8)$ & \\
Citalopram & $1(4)$ &
\end{tabular}

Abbreviations: CAPS, Clinician Administered PTSD Scale; TLEQ, Traumatic Life Events Questionnaire.

Mean \pm SD or N (\%).

$a_{p}<0.001$. one investigation conducted at 3T (Ham et al, 2007), all prior PTSD MRS hippocampus studies were performed at 1.5T, precluding reliable estimation of Glu.

This study tested the hypothesis that PTSD patients compared with trauma-exposed normal comparison (TENC) participants would show higher Glu concurrent with lower NAA in bilateral hippocampus, as well as elevations in the ratio of Glu/NAA that has been used as a marker of glutamate-related excitotoxicity in neurological illnesses. We also hypothesized that magnitude of metabolite alterations would be associated with severity of re-experiencing symptoms and trauma load in PTSD patients.

\section{MATERIALS AND METHODS}

\section{Participants}

We recruited 47 right-handed participants, 24 adults with PTSD and 23 TENC adults (ages 20-50) via advertisements in the local community. All participants provided written informed consent and the study was approved by the Institutional Review Board of McLean Hospital. Exclusion criteria were: (1) medical conditions that might affect brain structure; (2) current substance use disorder; (3) current nicotine dependence; (4) anxiolytic, anticonvulsant, mood stabilizing, or neuroleptic medication use within 4 weeks of the study; (5) history of substance abuse within the past 5 years; (6) lifetime history of substance dependence; (7) lifetime history of psychosis; (8) MR contraindications; (9) urine toxicology positive for psychoactive drugs or pregnancy. In addition, TENC participants had no current DSMIV Axis I disorder and no lifetime history of mood or anxiety disorder (including PTSD) based on the Structured Clinical Interview for DSM-IV Axis I disorders, Patient Edition (SCID-I/P; (First et al, 2002)). The groups were recruited to be individually matched on age and sex. Female participants were matched on menstrual cycle phase across groups, determined by self-report: six female PTSD and six TENC participants were in the follicular phase; five PTSD patients and four TENC were in the luteal phase; 3 PTSD patients and four TENC were post-menopausal. The TENC group had on average 1 year more of education (Table 1), although all participants had at least a high school education.

\section{Clinical Interviews and Self-Report Measures}

A doctoral-level psychologist (IMR) administered the SCID/ IP and the Clinician-Administered PTSD Scale (CAPS; (Blake et al, 1995)) to establish DSM-IV Axis-I diagnoses and PTSD symptom scores. PTSD participants reported as index traumas: childhood physical abuse $(n=5)$, childhood sexual abuse $(n=4)$, automobile or other violent accidents $(n=2)$, victim of physical assault $(n=7)$, combat exposure $(n=1)$, victim of sexual assault $(n=9)$, witnessing violent attack or abuse (3). One PTSD patient had current comorbid MDD, and five had past MDD. Three PTSD patients were taking a selective serotonin reuptake inhibitor (SSRI; stable dose for $\geqslant 8$ weeks), and receiving outpatient medication management. All other subjects were unmedicated.

Subjects were included in the TENC group if they endorsed at least one Criterion A trauma on the Traumatic Life Events Questionnaire (TLEQ; (Kubany et al, 2000)). 


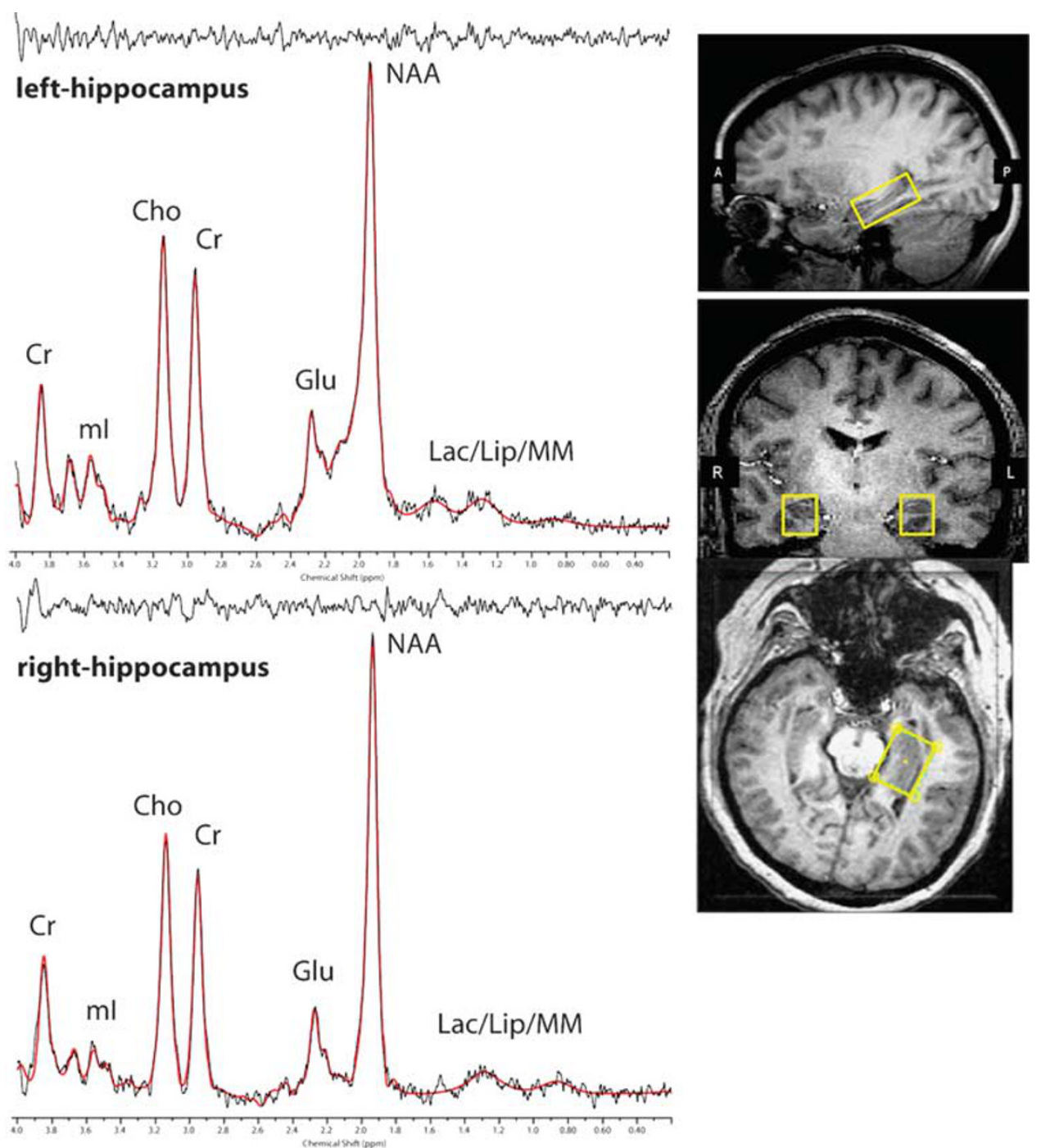

Figure I Three-plane view of hippocampus voxel placement and MRS spectral fit from a single participant.

TLEQ total score was used to define trauma load, specifically number of types of lifetime trauma types endorsed, for all participants.

\section{Magnetic Resonance Imaging and Spectroscopy}

Scanning was conducted on a $4.0 \mathrm{~T}$ Varian whole-body scanner (Agilent Technologies Inc., Santa Clara, CA), using a volumetric head coil. Head placement was confirmed for each participant using three-plane scout images. Following global shimming, high-contrast 3D fast low-angle shot T1weighted images were acquired to position a $1.5(\mathrm{~L}-\mathrm{R}) \times 2.0$ $(\mathrm{S}-\mathrm{I}) \times 3.0(\mathrm{~A}-\mathrm{P}) \mathrm{ml}$ voxel in the left and right hippocampi (Figure 1, inset). Voxels were obliqued and placed on the long axis of the hippocampus using the sagittal and axial planes. The anterior border of the voxel was positioned in the most anterior coronal slice that contained hippocampus but not amygdala, using the alveus and uncal recess to exclude amygdala tissue (for details, see Supplementary Information) (Pruessner et al, 2000; Manganas et al, 2007).
Manual voxel shimming produced linewidths that ranged from 8 to $12 \mathrm{~Hz}$. Spectral data were acquired using PointResolved Echo Spectroscopy Sequence modified for the current two-dimensional J-resolved MRS protocol (2DJPRESS) to collect 16 TE-stepped spectra in each voxel (Jensen et al, 2009). Echo times varied from 30 to $330 \mathrm{~ms}$ in $20 \mathrm{~ms}$ increments, which provided sufficient J-resolved bandwidth $(50 \mathrm{~Hz})$ to resolve Glu from Gln. Additional acquisition parameters included $\mathrm{TR}=2 \mathrm{~s}$, $\mathrm{f} 1$ acquisition bandwidth $=50 \mathrm{~Hz}$, spectral bandwidth $=2 \mathrm{kHz}$, readout duration $=512 \mathrm{~ms}, \mathrm{NEX}=16$, total scan duration $=8.5 \mathrm{~min}$. Unsuppressed water spectra were acquired immediately following metabolite acquisitions using the same 2DJPRESS sequence to collect 24 TE-stepped water scans with the parameters described above, but with 4 averages per TEstep. After data were acquired in one hippocampus voxel (total acquisition $=10.1 \mathrm{~min}$ ), the contralateral voxel (left or right hippocampus, counterbalanced across participants) was positioned and the same metabolite and water sequences repeated. Test-retest scans of four participants yielded good reproducibility (Supplementary Information). 
All spectroscopic data processing was performed using inhouse reconstruction code and LCModel fitting software (Provencher, 1993). In order to quantify proton metabolites, 16 TE-stepped free-induction decay series were zero-filled out to 64 points, Gaussian-filtered to minimize residual arising from NAA and Cr signals, and Fourier-transformed in the TE dimension. This resulted in 64J-resolved spectra over $50 \mathrm{~Hz}$. Using GAMMA-simulated J-resolved basis sets, every spectral extraction (bandwidth of $50 \mathrm{~Hz}$ ) was fit with its theoretically correct LCModel template (Jensen et al, 2009). The integrated area under the entire 2D surface for each metabolite was calculated by summing raw peak areas across all 64 J-resolved extractions. Figure 1 shows hippocampus spectra (black) and associated LCModel fitting (red) from a single participant. All spectra were visually inspected for fitting errors by the last author (JEJ); in addition, a full-width half maximum (FWHM) $\leqslant 12$ and signal-to-noise ratio $(\mathrm{SNR}) \geqslant 5$ were required for inclusion in the data analyses. Finally, spectra were excluded if either NAA or Glu had poorly fitted peaks, specifically: Cramer Rao Lower Bounds (CRLB) higher than 10\% for NAA, or higher than $20 \%$ for Glu. Based on these criteria, good quality datasets were available from 23 PTSD and 21 TENC for right hippocampus, and from 21 PTSD patients and 19 TENC for left hippocampus. Mean \pm standard deviation CRLBs were $3.5 \pm 1.3$ for Cr, $3.8 \pm 1.3$ for NAA, and $7.4 \pm 1.9$ for Glu. SNR did not differ significantly between PTSD and TENC participants in either left $\left(t_{38}=0.87, p=0.39\right)$ or right $\left(t_{42}=0.74, p=0.46\right)$ hippocampus, nor did FWHM (left: $t_{38}=0.45, p=0.66$; right: $\left.t_{42}=0.38, p=0.70\right)$. Unsuppressed water T2 values were derived for each voxel using TEstepped datasets and a least-squares algorithm, and these unsuppressed water values served as an internal concentration reference for metabolite/unsuppressed water $\left(\mathrm{H}_{2} \mathrm{O}\right)$ ratios. For metabolite/Cr ratios, the $\mathrm{Cr}$ denominator was the raw LCModel-outputted peak area for Cr. A single spectroscopist (JEJ) performed all MRS data acquisitions and spectral processing, blind to participant group assignment.

T1-weighted axial image sets were segmented into gray matter (GM), white matter (WM), and cerebrospinal fluid (CSF) binary-tissue maps (FSL, Oxford, UK). The proportion of gray matter (\%GM) in the left and right hippocampal voxels was calculated for each participant (Jensen et al, 2009; Silveri et al, 2013).

\section{Statistical Analyses}

NAA and Glu were examined normalized both to water $\left(\mathrm{H}_{2} \mathrm{O}\right)$ and to $\mathrm{Cr}$, to assess robustness of effects. In addition, Glu was normalized to NAA (Glu/NAA). Continuous variables were examined for skewness and kurtosis. All analyses statistically controlled for \%GM.

Group differences in NAA and Glu were examined using four repeated measures multivariate analyses of covariance (MANCOVA) with ratio type $\left(\mathrm{H}_{2} \mathrm{O}\right.$ or $\left.\mathrm{Cr}\right)$ as the withinsubject factor and the following dependent variables: 1) right NAA normalized to water and creatine (R_NAA/ $\mathrm{H}_{2} 0$, R_NAA/Cr); 2) left NAA normalized to water and creatine (L_NAA/ $\mathrm{H}_{2} \mathrm{O}, \mathrm{L} \_\mathrm{NAA} / \mathrm{Cr}$ ); 3 ) right Glu normalized to water and creatine (R_Glu/ $\left.\mathrm{H}_{2} 0, \mathrm{R} \_\mathrm{Glu} / \mathrm{Cr}\right) ; 2$ ) left Glu normalized to water and creatine (L_Glu/ $\left.\mathrm{H}_{2} 0, \mathrm{~L}_{-} \mathrm{Glu} / \mathrm{Cr}\right)$. Even though the groups were matched on age and sex, both were covariates to determine their contribution to variance in metabolite levels. Group differences in R_Glu/NAA and L_Glu/NAA were examined using analyses of covariance (ANCOVA), controlling for age and sex. Because of the substantive literature implicating lower hippocampus NAA in PTSD, the NAA results were not Bonferroni corrected. The MANCOVA and ANCOVA models predicting Glu ratios and Glu/NAA used a Bonferroni-corrected significance threshold of .025. Following significant multivariate omnibus effects, post hoc contrasts used Dunnett's test for multiple comparisons.

Bivariate correlations tested relationships of metabolite ratios with re-experiencing symptoms and trauma load. Only metabolites that differed significantly between groups underwent correlational testing (two-tailed $p<0.05$ ). Cohen's $d$ is reported as a measure of effect size (ES). All statistical analyses were conducted using JMP 9.0 (SAS Institute).

\section{RESULTS}

\section{Group Differences in NAA and Glu Metabolite Ratios}

Table 2 shows metabolite levels and between-group effect sizes. In the MANCOVA predicting right hippocampus NAA ratios, there was a significant main effect of group $\left(F_{(1,39)}=12.10, p=0.001\right)$. Post-hoc Dunnett's tests showed that both right $\mathrm{NAA} / \mathrm{H}_{2} \mathrm{O}(p=0.01)$ and right $\mathrm{NAA} / \mathrm{Cr}$ $(p=0.002)$ were significantly lower in PTSD than TENC participants. The MANCOVA predicting left hippocampus NAA ratios revealed a significant interaction of group $\mathrm{X}$ ratio $\left(F_{(1,35)}=4.64, p=0.04\right)$. Post-hoc Dunnett's showed that left $\mathrm{NAA} / \mathrm{H}_{2} \mathrm{O} \quad(p=0.02)$ but not left NAA/Cr $(p=0.35)$ was significantly lower in PTSD patients than TENC participants.

The MANCOVA predicting right hippocampus Glu ratios identified a significant main effect of group $\left(F_{(1,39)}=5.55\right.$, $p=0.02$ ), and post hoc Dunnett's showed that PTSD compared with TENC participants had significantly higher right $\mathrm{Glu} / \mathrm{H}_{2} \mathrm{O}(p=0.02)$ and right $\mathrm{Glu} / \mathrm{Cr}(p=0.05)$. In the MANCOVA with left hippocampus Glu ratios as dependent variables, neither the main effect of group nor the interaction of group by ratio was significant ( $p$ 's $>0.10)$.

In the ANCOVA predicting right hippocampus Glu/NAA, the group effect was significant $\left(F_{(1,38)}=8.85, p=0.005\right)$, reflecting significantly higher levels in PTSD than TENC participants. One PTSD participant had a R_Glu/NAA value higher than 3 SDs from the mean, and was excluded from the latter analysis. In the ANCOVA predicting left hippocampus Glu/NAA, the effect of group was not statistically significant.

All significant group differences were maintained when analyses were repeated without the three PTSD patients on a stable dose of SSRI. The effects of age and sex were not statistically significant $(p$ 's $>0.10)$ in any analysis.

\section{Correlations with Clinical Features}

Re-experiencing symptoms were negatively correlated with all NAA ratios that differed between the groups, namely with left $\mathrm{NAA} / \mathrm{H}_{2} \mathrm{O}(r=-0.46, n=21, p=0.04)$, right $\mathrm{NAA} / \mathrm{Cr}$ $(r=-0.55, n=23, p=0.006)$, and right NAA/ $\mathrm{H}_{2} \mathrm{O}(r=-0.35$, $n=23, p=0.10)$. There were positive correlations between re-experiencing symptoms and right hippocampus Glu ratios, statistically significant for $\mathrm{Glu} / \mathrm{H}_{2} \mathrm{O}(r=0.46, n=23$, 
$p=0.03)$ and not for Glu/Cr $(r=0.23, n=23, p=0.31)$. Finally, re-experiencing symptoms showed the largest correlation with right hippocampus Glu/NAA $(r=0.54$, $n=22, p=0.001$; Figure 2).

Trauma load was significantly positively correlated with right Glu/NAA in PTSD patients $\left(r_{\mathrm{s}}=0.52, p=0.01\right)$ but not TENC participants $\left(r_{\mathrm{s}}=-0.03, p=0.91\right)$. Trauma load was not significantly correlated with other metabolite ratios that differed between groups.

Because both re-experiencing symptoms and trauma load were significant correlates of right Glu/NAA in PTSD patients, we conducted a follow-up ANCOVA entering both as predictors of right $\mathrm{Glu} / \mathrm{NAA}$. In this analysis, re-experiencing symptoms $\left(F_{(1,17)}=5.27, p=0.04\right.$; variance inflation factor $(\mathrm{VIF})=1.12)$ but not trauma load $\left(F_{(1,17)}=1.94, p=0.18 ; \mathrm{VIF}=1.48\right)$ remained significantly associated with right hippocampus Glu/NAA, controlling for $\% \mathrm{GM}$, age, and sex.

\section{DISCUSSION}

Our data represent the first report of in vivo hippocampus glutamate metabolism measured with MRS in PTSD. We found that Glu and Glu/NAA levels were significantly higher in the right hippocampus of PTSD patients than traumaexposed comparison subjects. In addition, the neuron marker NAA was significantly lower in both left and right hippocampi of PTSD than TENC participants. Finally, levels of right hippocampus NAA and Glu scaled proportionally with severity of re-experiencing symptoms. Glu/NAA levels were correlated with both severity of re-experiencing symptoms and lifetime trauma load in PTSD patients, and when both correlates were entered as independent variables in the same regression only re-experiencing symptoms remained a significant predictor. These findings are consistent with those of prior MRS studies implicating compromised neuron integrity in the hippocampus of PTSD patients. They extend this literature by suggesting that lower NAA co-occurs with excess Glu and Glu/NAA in the right hippocampus, and that these metabolites may serve as clinically meaningful biomarkers of re-experiencing symptoms in PTSD.

Consistent with our hypotheses and the bulk of prior literature, NAA levels were significantly lower in left and right hippocampus of PTSD patients compared with TENC subjects. These findings remained statistically significant when three patients taking an antidepressant were excluded, indicating they were not a consequence of psychotropic drug effects. The neuron integrity findings in the right hippocampus were particularly robust because they were seen when NAA was normalized to either $\mathrm{H}_{2} \mathrm{O}$ or $\mathrm{Cr}$. In the left hippocampus, due to greater spectral data loss, we had sufficient power to detect as statistically significant an effect of $d=-0.80$ for left NAA/ $\mathrm{H}_{2} 0$, but not an effect of $d=-0.31$ for left NAA/Cr. Importantly, however, these effect sizes are similar in magnitude to those obtained by a meta-analysis of MRS investigations in PTSD (Karl and Werner, 2010): across studies of the hippocampus, larger effects were seen when left NAA was normalized to $\mathrm{H}_{2} \mathrm{O}(d=-0.63)$ than when it was normalized to $\mathrm{Cr}(d=-0.34)$. Thus, our pattern of results for hippocampus NAA ratios converges with prior

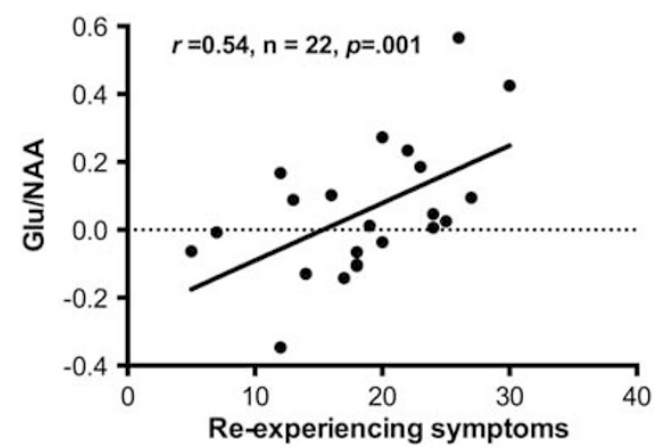

Figure 2 Correlations between re-experiencing symptoms of posttraumatic stress disorder and Glu/NAA in the right hippocampus, corrected for percent gray matter.

Table 2 Metabolite Levels ( $\mathrm{ml}$, Mean $\pm \mathrm{SD}$ ) in Right and Left Hippocampus Voxels on PTSD and TENC Participants

\begin{tabular}{|c|c|c|c|c|}
\hline Voxel & Metabolite & $\begin{array}{c}\text { PTSD } \\
(n=23)\end{array}$ & $\begin{array}{c}\text { TENC } \\
(n=2 I)\end{array}$ & $\begin{array}{c}\text { Effect sizel } \\
\text { p-value }\end{array}$ \\
\hline \multirow{6}{*}{$\begin{array}{l}\text { Right } \\
\text { hippocampus }\end{array}$} & $\mathrm{NAA} / \mathrm{H}_{2} \mathrm{O}$ & $1.05 \pm 0.22$ & $1.24 \pm 0.21$ & $-0.86 / .01$ \\
\hline & $\mathrm{NAA} / \mathrm{Cr}$ & $1.28 \pm 0.22$ & $\mid .51 \pm 0.22$ & $-1.03 / .002$ \\
\hline & $\mathrm{Glu} / \mathrm{H}_{2} \mathrm{O}$ & $0.89 \pm 0.17$ & $0.75 \pm 0.17$ & $0.79 / .01$ \\
\hline & Glu/Cr & $1.03 \pm 0.24$ & $0.88 \pm 0.24$ & $0.65 / .04$ \\
\hline & Glu/NAA ${ }^{a}$ & $0.82 \pm 0.17$ & $0.64 \pm 0.17$ & $1.07 / 0.002$ \\
\hline & Gray matter \% & $68.22 \pm 5.11$ & $65.10 \pm 6.18$ & $0.5 \mathrm{I} / \mathrm{NS}$ \\
\hline Voxel & Metabolite & $\begin{array}{c}\text { PTSD } \\
(n=2 I)\end{array}$ & $\begin{array}{c}\text { TENC } \\
(n=19)\end{array}$ & $\begin{array}{c}\text { Effect sizel } \\
\text { p-value }\end{array}$ \\
\hline \multirow{6}{*}{$\begin{array}{l}\text { Left } \\
\text { hippocampus }\end{array}$} & $\mathrm{NAA} / \mathrm{H}_{2} \mathrm{O}$ & $1.01 \pm 0.24$ & $1.20 \pm 0.24$ & $-0.80 / 0.02$ \\
\hline & $\mathrm{NAA} / \mathrm{Cr}$ & $1.07 \pm 0.21$ & $1.14 \pm 0.21$ & $-0.3 \mathrm{I} / \mathrm{NS}$ \\
\hline & $\mathrm{Glu} / \mathrm{H}_{2} \mathrm{O}$ & $0.78 \pm 0.17$ & $0.84 \pm 0.18$ & $-0.34 / \mathrm{NS}$ \\
\hline & $\mathrm{Glu} / \mathrm{Cr}$ & $0.82 \pm 0.17$ & $0.82 \pm 0.20$ & $-0.01 / \mathrm{NS}$ \\
\hline & Glu/NAA ${ }^{b}$ & $0.80 \pm 0.19$ & $0.72 \pm 0.20$ & $0.31 / \mathrm{NS}$ \\
\hline & Gray matter \% & $68.10 \pm 4.32$ & $68.84 \pm 4.64$ & $-0.17 / \mathrm{NS}$ \\
\hline
\end{tabular}

General linear models required a significant omnibus test and values are adjusted for percent gray matter in the voxel. Effect size is Cohen's $d$.

${ }^{a}$ One PTSD excluded as statistical outlier.

bOne PTSD and one TENC excluded as statistical outliers.

MRS reports of bilateral hippocampus NAA deficits in PTSD (Ham et al, 2007; Mahmutyazicioglu et al, 2005; Neylan et al, 2003; Schuff et al, 2001, 2008; Shu et al, 2013).

Elevations of Glu and Glu/NAA serve as biomarkers of glutamate excitotoxicity in neurological disorders, and this is the first report that MRS detects similar alterations in the hippocampus of PTSD patients. We found that right but not left hippocampus Glu and Glu/NAA ratios were significantly higher in PTSD than TENC participants. Moreover, the group difference in right hippocampus Glu/NAA was the largest between-group effect size seen in this study (Cohen's $d=1.07$; Table 2). The group differences in left hippocampus Glu and Glu/NAA corresponded to smaller effect sizes that did not reach statistical significance. At the same time, the 
extant literature provides precedent for more severe right than left hippocampus pathology in PTSD. For instance, a meta-analysis of hippocampus morphometric MRI studies concluded that right hippocampus volume, but not left or total hippocampus volume, is significantly smaller in PTSD than TENC participants (Woon et al, 2010). All told, because the current study is the first to report on hippocampus Glu indices in PTSD, additional MRS investigations will be needed to determine conclusively their nature and laterality.

A number of cellular and metabolic changes could lead to higher than normal Glu and Glu/NAA. Most Glu is synthesized from Gln within neurons, then released into extracellular space where it binds to post-synaptic receptors, until its actions are terminated by its re-uptake into astrocytes. Within astrocytes, Glu is converted to Gln, which is transported back to the pre-synaptic neuron and recycled back into Glu (Rothman et al, 1999). Animal models support an association of chronic stress or repeated trauma with decreased reuptake of Glu into astrocytes (Banasr et al, 2010), higher basal Glu release, and prolonged stimulus-evoked Glu release from hippocampal neurons (Popoli et al, 2012). These processes all lead to Glu elevation, which in turn can initiate a cascade of cellular processes that culminates in neuron injury. Although our cross-sectional study limits interpretations regarding disease severity and cannot determine which metabolite alteration occurred first, future longitudinal studies could assess whether higher Glu predates lower NAA, as would be predicted by an excitotoxicity hypothesis.

We found significant associations of PTSD re-experiencing symptoms with hippocampus NAA and Glu ratios. More severe re-experiencing was significantly negatively correlated with all three left and right NAA ratios that differed between groups. Re-experiencing symptom severity also showed significant positive correlations with $\mathrm{Glu} / \mathrm{H}_{2} \mathrm{O}$ and $\mathrm{Glu} /$ NAA in the right hippocampus. These findings add to a scant literature on clinical correlates of hippocampus MRS measures in PTSD. For Glu, they are the first evidence of relevance to clinical phenomenology, buttressing other research on hippocampus glutamate metabolism and behavioral phenotypes of PTSD (eg, learning and memory; Popoli et al, 2012). For NAA, multiple studies previously had found no significant association with PTSD severity (reviewed in Karl and Werner, 2010), and some had found that greater hippocampus NAA deficits predicted more severe symptoms (Brown et al, 2003; Neylan et al, 2003; Shu et al, 2013). Importantly, most prior studies had examined total symptom severity but not groupings of symptoms more likely to share biological underpinnings. Re-experiencing symptoms involve recalling emotionally traumatic events, and two earlier studies found a relationship of these intrusive experiences with lower hippocampus NAA (Brown et al, 2003; Shu et al, 2013). Similarly, using morphometric MRI, PTSD reexperiencing symptoms have been found to correlate with lower hippocampal volume (Lindauer et al, 2006; Villarreal et al, 2002, but see Nakano et al, 2002). Our findings add to this literature by providing evidence of an association between intrusive symptoms and hippocampus Glu metabolism in PTSD.

Lifetime trauma load was associated with significantly higher right hippocampus Glu/NAA in PTSD patients. This finding presents the possibility that Glu/NAA abnormalities partly reflect neurotoxic effects of trauma, perhaps occurring above a certain magnitude of trauma load since the relationship was seen in PTSD but not TENC participants. However, this hypothesis requires testing using a longitudinal design. Moreover, enthusiasm for this interpretation is tempered by the finding that the association between trauma load and right hippocampus Glu/NAA loses its significance when controlling for the significant correlation with re-experiencing symptoms. This latter finding suggests that higher Glu/NAA levels are indicators of disease processes more so than trauma vulnerability factors. It is also possible that trauma load synergized with differential presence of other risk factors, such as genetic and epigenetic factors that affect differential risk for PTSD subsequent traumatic life events (Gilbertson et al, 2002; Ressler et al, 2011). Future studies of samples more homogeneous regarding types of trauma and genetic/epigenetic risk factors, will help parse the heterogeneous etiopathophysiology of PTSD.

This study has several limitations to be considered in its interpretation. First, the sample size limited our ability to detect as significant some of the small-to-medium effects. Second, although all our comparison subjects had experienced at least one major trauma, on average they reported a lower total number of lifetime traumas than PTSD patients, a limitation that is difficult to avoid when recruiting healthy (asymptomatic) trauma-exposed participants. Lower variance of trauma load may have precluded detection of significant correlations with MRS metabolites in the TENC group. Third, current MRS methods detect total brain Glu concentration averaged over an area of interest that includes both intracellular and extracellular compartments of brain Glu. Thus, we cannot discern whether higher right hippocampus Glu reflects higher vesicular or neurotransmitter pools, or both. Finally, the voxel size needed for sufficient SNR to parse Glu and Gln may have resulted in some partial volume effects from the parahippocampal gyrus (also see (Brown et al, 2003; Freeman et al, 1998)).

In summary, the picture that emerges from our pattern of findings is that glutamate excess and neuron compromise may be concurrent pathophysiologic mechanisms in the right hippocampus of people with PTSD. The clinical significance of these hippocampus abnormalities is affirmed by their robust correlations with severity of re-experiencing symptoms. Future longitudinal research should examine whether higher Glu predates and predicts lower NAA, and whether altered Glu and Glu/NAA might serve as biomarkers of disease progression and treatment response. If so, these MRS metabolites could become tools for guiding personalized treatment selection or monitoring treatment response in PTSD, ultimately playing a similar clinical decision-making role as they do for certain neurological disorders (Burtscher and Holtas, 2001; Mason and Krystal, 2006).

\section{FUNDING AND DISCLOSURE}

This research was supported by a grant from the Dana Foundation (Brain and Immuno-Imaging Program; IMR). Dr Rosso received partial salary support from the National Institute of Health (NIH/NIMH R01 MH096987; IMR). Drs Silveri and Jensen have current and past funding from the $\mathrm{NIH}$, and Dr Rauch from the NIH and US Army Military Operational Medicine Research Program. The authors declare no conflict of interest. 


\section{REFERENCES}

Adamec R, Hebert M, Blundell J, Mervis RF (2012). Dendritic morphology of amygdala and hippocampal neurons in more and less predator stress responsive rats and more and less spontaneously anxious handled controls. Behav Brain Res 226: 133-146.

Banasr M, Chowdhury GMI, Terwilliger R, Newton SS, Duman RS, Behar KL et al (2010). Glial pathology in an animal model of depression: reversal of stress-induced cellular, metabolic and behavioral deficits by the glutamate-modulating drug riluzole. Mol Psychiatry 15: 501-511.

Blake DD, Weathers FW, Nagy LM, Kaloupek DG, Gusman FD, Charney DS et al (1995). The development of a clinicianadministered PTSD scale. J Trauma Stress 8: 75-90.

Bremner D, Mletzko T, Welter S, Siddiq S, Reed L, Williams C et al (2004). Treatment of posttraumatic stress disorder with phenytoin: an open-label pilot study. J Clin Psychiatry 65: 1559-1564.

Bremner JD, Randall P, Scott TM, Bronen RA, Seibyl JP, Southwick SM et al (1995). MRI-based measurement of hippocampal volume in patients with combat- related posttraumatic stress disorder. Am J Psychiatry 152: 973-981.

Brown S, Freeman T, Kimbrell T, Cardwell D, Komoroski R (2003). In vivo proton magnetic resonance spectroscopy of the medial temporal lobes of former prisoners of war with and without posttraumatic stress disorder. J Neuropsychiatry Clin Neurosci 15: 367-370.

Burtscher IM, Holtas S (2001). Proton MR spectroscopy in clinical routine. J Magn Reson Imaging 13: 560-567.

Chambers RA, Bremner JD, Moghaddam B, Southwick SM, Charney DS, Krystal JH (1999). Glutamate and post-traumatic stress disorder: toward a psychobiology of dissociation. Semin Clin Neuropsychiatry 4: 274-281.

Doelken MT, Mennecke A, Stadlbauer A, Kecskeméti L, Kasper BS, Struffert T et al (2010). Multi-voxel magnetic resonance spectroscopy at $3 \mathrm{~T}$ in patients with idiopathic generalised epilepsy. Seizure 19: 485-492.

First MB, Spitzer RL, Gibbon M, Williams JBW (2002). Structured Clinical Interview for DSM-IV-TR Axis I Disorders, Patient Edition (SCID-I/P). Biometrics Research, New York State Psychiatric Institute: New York, USA.

Freeman TW, Cardwell D, Karson CN, Komoroski RA (1998). In vivo proton magnetic resonance spectroscopy of the medial temporal lobes of subjects with combat-related posttraumatic stress disorder. Magn Reson Med 40: 66-71.

Gilbertson MW, Shenton ME, Ciszewski A, Kasai K, Lasko NB, Orr SP et al (2002). Smaller hippocampal volume predicts pathologic vulnerability to psychological trauma. Nat Neurosci 5: 1242-1247.

Ham B-J, Chey J, Yoon SJ, Sung Y, Jeong D-U, Ju Kim S et al (2007). Decreased N-acetyl-aspartate levels in anterior cingulate and hippocampus in subjects with post-traumatic stress disorder: a proton magnetic resonance spectroscopy study. Eur J Neurosci 25: 324-329.

Helms G, Ciumas C, Kyaga S, Savic I (2006). Increased thalamus levels of glutamate and glutamine (Glx) in patients with idiopathic generalised epilepsy. J Neurol Neurosurg Psychiatry 77: 489-494.

Jensen JE, Licata SC, Ongur D, Friedman SD, Prescot AP, Henry ME et al (2009). Quantification of J-resolved proton spectra in two-dimensions with LCModel using GAMMAsimulated basis sets at 4 Tesla. NMR Biomed 22: 762-769.

Jing $H$, Lin $M$ (2010). Study of the features of proton MR spectroscopy (1H-MRS) on amyotrophic lateral sclerosis. J Magn Reson Imaging 31: 305-308.

Karl A, Schaefer M, Malta LS, Dorfel D, Rohleder N, Werner A (2006). A meta-analysis of structural brain abnormalities in PTSD. Neurosci Biobehav Rev 30: 1004-1031.

Karl A, Werner A (2010). The use of proton magnetic resonance spectroscopy in PTSD research-Meta-analyses of findings and methodological review. Neurosci Biobehav Rev 34: 7-22.
Kitayama N, Vaccarino V, Kutner M, Weiss P, Bremner JD (2005). Magnetic resonance imaging (MRI) measurement of hippocampal volume in posttraumatic stress disorder: A meta-analysis. J Affect Disord 88: 79-86.

Krystal JH, Karper LP, Seibyl J, Freeman G, Delaney R, Bremner J et al (1994). Subanesthetic effects of the noncompetitive NMDA antagonist, ketamine, in humans: Psychotomimetic, perceptual, cognitive, and neuroendocrine responses. Arch Gen Psychiatry 51: 199-214.

Kubany ES, Haynes SN, Leisen MB, Owens JA, Kaplan A, Watson SB et al (2000). Development and preliminary validation of a brief broad-spectrum measure of trauma exposure: the Traumatic Life Events Questionnaire. Psychol Assess 12: 210-224.

Lewerenz J, Maher P (2015). Chronic glutamate toxicity in neurodegenerative diseases-What is the evidence? Front Neurosci 9: 469.

Lindauer RJL, Olff M, Van Meijel EPM, Carlier IVE, Gersons BPR (2006). Cortisol, learning, memory, and attention in relation to smaller hippocampal volume in police officers with posttraumatic stress disorder. Biol Psychiatry 59: 171-177.

Mahmutyazicioglu K, Konuk N, Ozdemir H, Atasoy N, Atik L, Gundogdu S (2005). Evaluation of the hippocampus and the anterior cingulate gyrus by proton MR spectroscopy in patients with post-traumatic stress disorder. Diagn Interv Radiol 11: 125-129.

Manganas LN, Zhang X, Li Y, Hazel RD, Smith SD, Wagshui ME et al (2007). Magnetic resonance spectroscopy identifies neural progenitor cells in the live human brain. Science 318: 980-985.

Mark LP, Prost RW, Ulmer JL, Smith MM, Daniels DL, Strottmann JM et al (2001). Pictorial review of glutamate excitotoxicity: Fundamental concepts for neuroimaging. Am J Neuroradiol 22: 1813-1824.

Mason GF, Krystal JH (2006). MR spectroscopy: Its potential role for drug development for the treatment of psychiatric diseases. NMR Biomed 19: 690-701.

McEwen BS (2006). Protective and damaging effects of stress mediators: Central role of the brain. Dialogues Clin Neurosci 8: 367-381.

McEwen BS (2007). Physiology and neurobiology of stress and adaptation: Central role of the brain. Physiol Rev 87: 873-904.

McEwen BS, Magarinos AM (1997). Stress effects on morphology and function of the hippocampus. Ann NY Acad Sci 821: 271-284.

Nakano T, Wenner M, Inagaki M, Kugaya A, Akechi T, Matsuoka Y et al (2002). Relationship between distressing cancer-related recollections and hippocampal volume in cancer survivors. Am J Psychiatry 159: 2087-2093.

Neylan TC, Schuff N, Lenoci M, Yehuda R, Weiner MW, Marmar CR (2003). Cortisol levels are positively correlated with hippocampal N-acetylaspartate. Biol Psychiatry 54: 1118-1121.

Popoli M, Yan Z, McEwen BS, Sanacora G (2012). The stressed synapse: the impact of stress and glucocorticoids on glutamate transmission. Nat Rev Neurosci 13: 22-37.

Provencher SW (1993). Estimation of metabolite concentrations from localized in vivo proton NMR spectra. Magn Reson Med 30: 672-679.

Pruessner JC, Li LM, Serles W, Pruessner M, Collins DL, Kabany N et al (2000). Volumetry of hippocampus and amygdala with highresolution MRI and three-dimensional analysis software: minimizing the discrepancies between laboratories. Cereb Cortex 10: 433-442.

Rauch SL, Shin LM, Phelps EA (2006). Neurocircuitry models of posttraumatic stress disorder and extinction: human neuroimaging research-past, present, and future. Biol Psychiatry 60: 376-382.

Ressler KJ, Mercer KB, Bradley B, Jovanovic T, Mahan A, Kerley K et al (2011). Post-traumatic stress disorder is associated with PACAP and the PAC1 receptor. Nature 470: 492-497. 
Rothman DL, Sibson NR, Hyder F, Shen J, Behar KL, Shulman RG (1999). In vivo nuclear magnetic resonance spectroscopy studies of the relationship between the glutamate-glutamine neurotransmitter cycle and functional neuroenergetics. Philos Trans $R$ Soc Lond B Biol Sci 354: 1165-1177.

Sapolsky RM (1996). Why stress is bad for your brain. Science 273: 749-750.

Savic I, Thomas AM, Ke Y, Curran J, Fried I, Engel J (2000). In vivo measurements of glutamine+glutamate (Glx) and $\mathrm{N}$-acetyl aspartate (NAA) levels in human partial epilepsy. Acta Neurol Scand 102: 179-188.

Schuff N, Neylan TC, Fox-Bosetti S, Lenoci M, Samuelson KW, Studholme C et al (2008). Abnormal N-acetylaspartate in hippocampus and anterior cingulate in posttraumatic stress disorder. Psychiatry Res Neuroimag 162: 147-157.

Schuff N, Neylan TC, Lenoci MA, Du AT, Weiss DS, Marmar CR et al (2001). Decreased hippocampal $\mathrm{N}$-acetylaspartate in the absence of atrophy in posttraumatic stress disorder. Biol Psychiatry 50: 952-959.

Shu XJ, Xue L, Liu W, Chen FY, Zhu C, Sun XH et al (2013). More vulnerability of left than right hippocampal damage in righthanded patients with post-traumatic stress disorder. Psychiatry Res 212: 237-244.
Silveri MM, Sneider JT, Crowley DJ, Covell MJ, Acharya D, Rosso IM et al (2013). Frontal lobe gamma-aminobutyric acid levels during adolescence: associations with impulsivity and response inhibition. Biol Psychiatry 74: 296-304.

Smith ME (2005). Bilateral hippocampal volume reduction in adults with post-traumatic stress disorder: a meta-analysis of structural MRI studies. Hippocampus 15: 798-807.

Srinivasan R, Sailasuta N, Hurd R, Nelson S, Pelletier D (2005). Evidence of elevated glutamate in multiple sclerosis using magnetic resonance spectroscopy at 3T. Brain 128: 1016-1025.

Tisell A, Leinhard OD, Warntjes JBM, Aalto A, Smedby Ö, Landtblom AM et al (2013). Increased concentrations of glutamate and glutamine in normal-appearing white matter of patients with multiple sclerosis and normal MR imaging brain scans. PLoS ONE 8: e61817

Villarreal G, Hamilton DA, Petropoulos H, Driscoll I, Rowland LM, Griego JA et al (2002). Reduced hippocampal volume and total white matter volume in posttraumatic stress disorder. Biol Psychiatry 52: 119-125.

Woon FL, Sood S, Hedges DW (2010). Hippocampal volume deficits associated with exposure to psychological trauma and posttraumatic stress disorder in adults: A meta-analysis. Prog Neuropsychopharmacol Biol Psychiatry 34: 1181-1188.

Supplementary Information accompanies the paper on the Neuropsychopharmacology website (http://www.nature.com/npp) 